\title{
7. USE OF STANDARD/SIMPLIFIED GRAPHIC LANGUAGE FOR DEVELOPMENT: A COMMUNICATION CHALLENGE
}

\author{
Sylvester Zifegha Ebigbagha ${ }^{232}$
}

\begin{abstract}
The decision to use standard, or simplified graphic language for development communication is usually problematic as a result of target audience conditions in developing countries that make presentation of a full range of development messages with uniformity across cultural delineations difficult. A rash decision often result in production of ineffective media with grave consequences for structural and human growth. Therefore, this paper focused on the communication challenge of utilizing standard/simplified graphic language for advancement. It employed a triangulation of the Critical-Historical-Analytic examination, and Content analysis methods. The paper started with the need to make informed decision on the use standard/simplified graphic language appropriate for receivers. Furthermore, it examined the role/activities of communication actors in campaign situation using the Shuttle models. Moreover, it considered target audience conditions with particular attention to illiteracy, and visual perception across cultural boundaries. And it evidenced the deployment of standard/simplified graphic language for campaigns in Africa. The paper found that target audience pretest is the panacea for decisive use of standard/simplified graphic language appropriate for target audience; and ended with the need to adopt participatory communication practices that afford its realization.
\end{abstract}

Key words: Communication challenge, Development initiatives, Audience conditions, Use of standard or simplified graphic language, Target audience evaluation

\section{Introduction}

Every nation across the world embarks upon human development programmes for growth, and advancement that culminate in improved standard of living. Towards this end, graphic media is usually employed to inform, persuade and mobilize target audience to support development efforts. So, graphic communication is indispensable for the success of development initiatives.

However, most of the development programmes, particularly in Africa, are initiated by developed funding nations or sources. Examples include United States Agency for International Development (USAID), and United Kingdom Department for International Development (DFID), to mention a few. These funded initiatives, are usually accompanied with packages of instructions, information, and development messages. According to Ejembi (1989:109), this "information package is seldom sufficiently simplified to meet target audience requirements of developing nations, which create pressure on communicators in such nations to simplify".

Simplifying messages to afford receivers' cultural and educational needs as well as cognitive abilities is needful because different cultures produce different pictures as environmental experiences differ. In addition, knowledge about the

232 Professor PhD., Niger Delta University from Wilberforce Island, Bayelsa State, Nigeria, email: ebigbaghazsylvester@yahoo.com 
conventions that guide the creation of a given set of pictures affect how the pictures are perceived. Even though it is relatively challenging to find the appropriate picture for a target audience with unknown abilities of pictorial perception, graphic messages still need be structured in accordance with target audience knowledge, attitude, and practices. This could be achieved through collaboration of all communication actors including the graphic encoder and the receivers.

The above is often made difficult because of prevalent adoption of the Modernization paradigm of development that employs the top-down communication practices. This practice is usually characterized by the source control of the entire communication development process, whereby information flow is from above and outside, which largely undermines the proper intervention of target audience and the graphic encoder (Ebigbagha, 2016). So, the predominant directionality of development information and ideas are foreign, abstract, and standard, which in most cases need be adapted/simplified into graphic language (a mode, which development message is transformed) suitable for target audience in the recipient countries.

From the foregoing, a key challenge is the decision to use standard graphic language (graphic messages supplied, based on recognition of its excellence by the source or media requesting agency) or simplified graphic language (graphic message made simple to afford processing by target audience, even with lowlevel or non-existent communication skills). The resolution of this dilemma is critical to development communication actors, particularly the graphic encoder (who is assigned the responsibility to transform development ideas into graphic messages for effective communication). This is because a rash decision made in the process of encoding development ideas into graphic language, usually results in the production of ineffective graphic media with dire consequences for human development.

Making informed decision on the use of graphic language appropriate for target audience information processing and desired response, often requires the participation of all stakeholders of communication (Source, Media team members - with particular emphasis on the graphic encoder, and target audience) to sufficiently and necessarily perform their roles. In this collaborative effort, the graphic encoder should actively intervene in all stages of the media production process. Where this level of involvement is not possible as a result of logistical reasons, the graphic encoder need be embedded in at least, the Clarification with source's aim and target audience, topic study, pretest, and evaluation stages of the media production process (Ebigbagha, 2020). This allows for adequate consideration of target audience conditions that potently impact on graphic encoding choices (stimulus conditions), and target audience perception of and response to a given graphic message.

The taking of target audience conditions into consideration in making appropriate graphic language, has immense implication for graphic communication. For example, in Africa, it is a common knowledge that the audience condition is characterized by cross-cultural variables, and prevalence 
ignorance, superstition and illiteracy (Obionu, 2001). The consideration of these factors in development communication is crucial to making efficient and effective graphic encoding choices that matches the knowledge, attitude and practices of target audience. This requires understanding of the roles of communication actors in campaign situation, target audience conditions, and the use of standard/simplified graphic language in development communication. Towards this end, the Shuttle models would be examined to guide our discussion on roles of communication actors during campaign. Also, target audience conditions would be examined by consideration of the implications of illiteracy, and crosscultural variables that impact on target audience perception of graphic messages. And evidence of the challenge of using standard/simplify graphic language for development would be presented with examples from health campaigns in Egypt and Nigeria.

\section{Roles of Communication Actors on Graphic Language}

The source, graphic encoder, and target audience perform a set of interrelated and complex activities, functions and roles in the process of developing graphic language at the preparatory and advance stages of a graphic communication campaign. Examples include decoding, interpreting, and encoding development messages. However, in order to avoid ambiguity in discussing these roles of communication actors in campaign situation, the Shuttle models lend themselves as a veritable guide.

The Shuttle Model of Graphic Communication Campaign I and II

The Shuttle Models of Graphic Communication Campaign I and II (figure 1a and 1b) described/prescribed the activities and roles of the source/graphic encoder and target audience in a communication campaign. It shows that the roles and activities of communication actors on messages at the preparatory stage of a campaign, Model I (figure 1a), are different from the advance stage, Model II, (figure 1b). The activities on the development messages are shown in the communication campaign arrow, which shuttles from model I to model II forward and backward carrying along variables that are used for analysing specific/general communication situations. The models identified three components under which the graphic communication campaign are examined, these are: (i) Source, (ii) Communication campaign; and (iii) Receiver. Also, the activities/roles, which take place under these components, are classified into three as follows: (i). Function (ii) Modality; and (iii) Targets. In the preparatory stage of the campaign, Model I (figure 1a), a detail analysis of activities/roles are given as follows:

(i) Function, is concerned with the role played by the three components in the campaign process as follows:

a. Source: performs the role of an encoder, as development messages are transformed into media. Also, Source performs the role of an interpreter, as response from the receiver is interpreted. And Source performs the role of a decoder, as development messages are decoded through feedback.

b. Communication campaign: concerns the messages, which could be persuasive 
or informative. This is sent to the receiver, whose response gets to the source through the feedback loop.

c. Receiver: performs the role of decoder, and an interpreter as messages received are decoded and interpreted respectively. This forms the basis for the response the source receives through the feedback loop in the campaign arrow.

(ii) Modality, is concerned with the nature, and treatment of the graphic messages to be communicated, in order to achieve the specific/general objectives of the communication campaign. These are located in the head of the campaign arrow as follows:

a. Pragmatics: deals with how words and phrases are used with special meanings in particular situation. This is necessary because words and phrases carry different meanings/interpretations in different context.

b. Aesthetics: is about how beautiful the graphic language is in appearance. This is necessary in order for the message to attract attention and arouse interest.

c. Semantics: deals with the meaning of words. This is necessary in order to ensure that words convey meanings as intended. The source's neglect of the fact that a sent message may produce different meanings to the receiver is a common reason for communication failure (McQuail and Windahl, 1986).

d. Syntax: is concerned with how the graphic and typographic elements are ordered and connected in the message. This is important in order to facilitate comprehension and the decoding sequence of the message. The necessity of this is brought to the fore when we realise that target audience who have not learn to read and write, do not usually look at pictures in the order intended. Therefore, it often proves helpful as messages are being tested to ask sample of the target audience to arrange the graphic and typographic variables in a message into sequence that seem more logical to the target audience (Ejembi, 1989).

e. Style: is about how the message is shaped to assume a particular outlook. The appearance, the relationship between the typographic and graphic elements contributes to detection, attraction, interest, etc., necessary to achieve the overall desired communication goal.

(iii) Targets, are about the specific and general objectives of the communication campaign, which the campaign arrow aims at achieving.

In the advance stage of the communication campaign, Model II (figure 1b), a detail analysis of activities and roles are described/prescribed as follows:

(i) Function, is as described in Model I (figure 1a)

a. Source: is as described in Model I (figure 1a)

b. Communication campaign: it is concerned with roles/activities that are different from the ones in the preparatory stage (Model I). The difference is in the activities/roles in the campaign arrow. After the elements in the campaign arrow in Model I has been considered, the graphic encoder proceeds to the activities/roles in the campaign arrow in Model II as follows:

i. Consult: consultations are made on the topic study with subject matter specialists in order to have a better understanding of the topic study and appropriately identify and match user-needs with source's ideas. 
ii. Review: it is concerned with the careful examination of the knowledge yielding materials on the message. This is necessary in order for the graphic encoder to have a comprehensive view of the whole situation that would enhance the production of a more effective graphic language. Often, information from the review impact on the inductive activity during the creative process and result in a better design solution.

iii. Encode: it is the process of transforming the reviewed information about development messages into graphic language, using graphic and typographic elements. It is the activity/role of the graphic encoder.

iv. Pre-test: is the exposure of the encoded media draft to a sample of the target audience in order to test its effectiveness so that it can be developed further.

v. Evaluate: assessment of the result of the pre-test; and

vi. Recode: is to make amendments on the media if necessary, this need be hinged on the pre-test results.

c. Receiver: is as described in Model I (figure 1a).

(ii) Modality, is about how the finally encoded graphic message is distributed to target audience.

These include:

a. Inform: it is the activity carried out to formally declare to target audience the development programme. This could be done through electronic media or print media to mention a few.

b. Advertise: concerns the activities embarked upon to make known the content of the development message, which could be the change of ideas, attitudes or innovations to be adopted/utilized; and

c. Publicize: is concerned with activities to make known the information about specific goals or objectives within the overall aim of the development initiative.

(iii) Targets, it is as described in Model I (figure 1a).

A common component in both Models I and II, is the socio-cultural environment, within which the whole graphic communication campaign process operates. This underscores the socio-cultural environment as an indispensable variable in decision-making to determine the use of standard or simplified graphic language that would be suitable to a given target audience. Invariably, it is pivotal for success in achieving the overall aim of the graphic communication campaign.

\section{Implications of the Shuttle Models}

The shuttle models are important to the graphic encoder and other members of the media team in development communication. These described/prescribed activities and roles of communication actors on the media development process at the preparatory and advance stages of a graphic communication campaign. As communication actors perform their roles properly, the decision on using the appropriate graphic language for a given target audience would be achieved with desired outcome.

It classified activities/roles of the source, message and receiver into three: function, modality and targets. The source performs the role of encoder, interpreter and decoder, while the receiver performs the role of decoder and interpreter of messages, and response gets back to the source through feedback. 
While these functions remain the same for the source and the receiver at both stages of the campaign, it is different for the message, which is located in the campaign arrow.

In the preparatory stage, roles and activities are concerned with how to ensure that the message is as informative or persuasive as possible. Particular attention is given to the nature and treatment of the graphic message, whereby, the modality of pragmatics, aesthetics, semantics, syntax and style are critically examined. While in the advance stage, roles and activities are oriented to consult, review, encode, pre-test, evaluate, and recode drafted graphic messages (where necessary). In addition, it is concerned with the modality to distribute the encoded graphic messages to target audience through information, advertising and publicity.

In both stages the achievement of the specific and general objectives of the campaign is the target of all roles and activities in the campaign arrow. And the socio-cultural environment is taken into account, which is an important factor for the success of development communication campaign. The knowledge of the functions of the source and receiver as well as the change of roles/activities on the message at the preparatory and advance stages of the campaign, is important to the graphic encoder in particular and the media team in general. This affords proper performance of roles/activities that prevents obtrusive intrusions, which often impact negatively on graphic language in the development process.

However, do all graphic communication campaign need pass through the stages, roles and activities presented in the shuttle campaign models? The prevalent communication practice in development campaigns, the hierarchical/Top-down approach in Nigeria, for example, ignores many of these steps, activities and roles presented in the models. The graphic encoder and the target audience are often neglected in the decision-making process during communication development.

Nevertheless, it is an essential model to providing a guide that would facilitate the collaborative efforts between the graphic encoder and other members of the media team as well as other stakeholders in development communication for informed decision-making on utilising appropriate graphic messages. This has become very necessary in the participatory approach that now coexist with the prevalent hierarchical, whereby collaboration between all communication actors in the communication process is indispensable to generate suitable graphic language that afford target audience perception and desired responses.

The above requires all stakeholders to actively intervene in the process of communication development but have different levels of experience that do not permit equal level of participation in decision making that concerns them. While the funding source is usually literate and developed, the target audience are largely non-literate, with plurality of cross-cultural variables and almost nonexistent skills of communication. This target audience conditions is a formidable challenge that impact on the use of standard or simplified graphic language. 


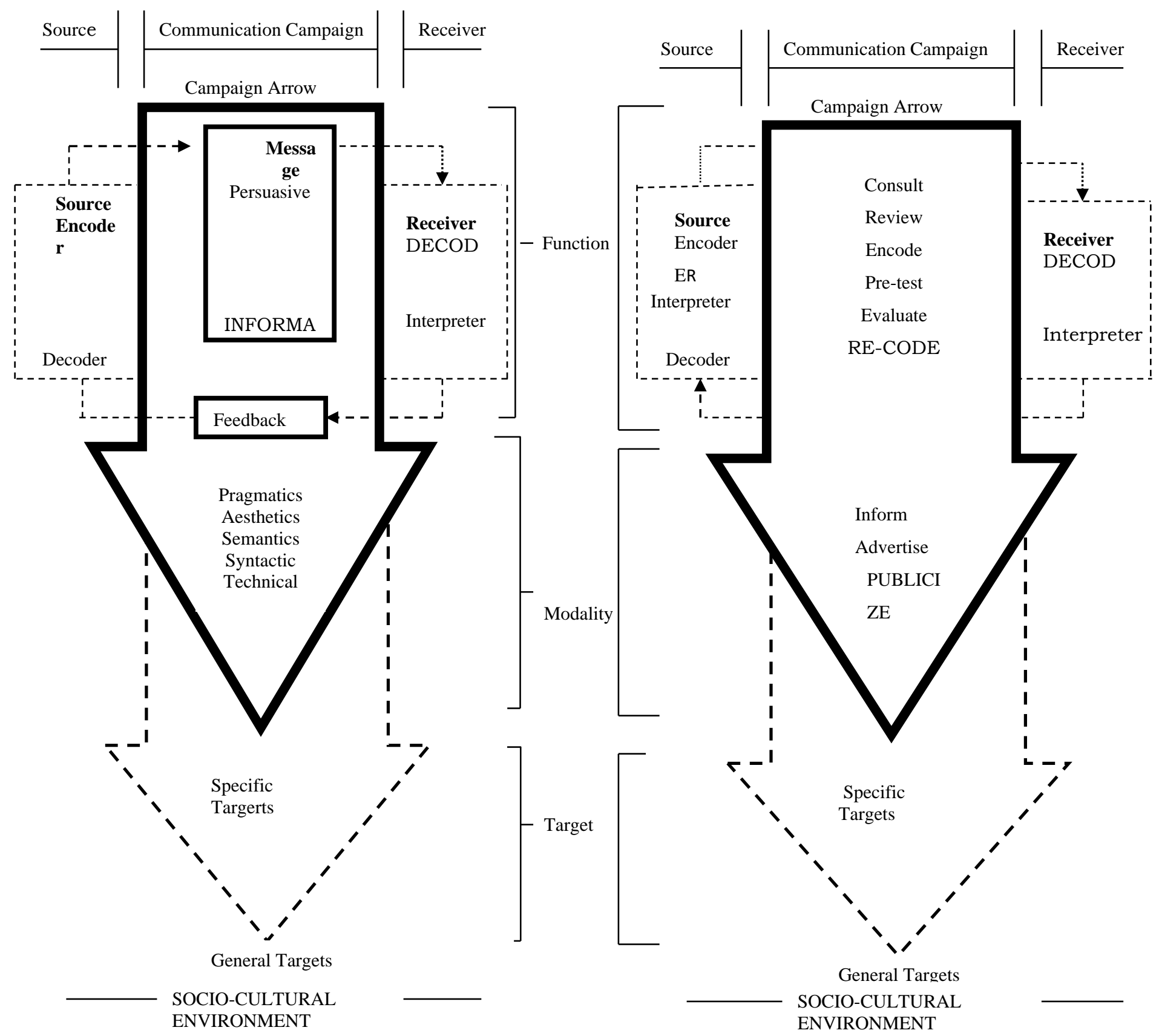

Figure 1a: The Shuttle Campaign Model I

(Ejembi, 1989)

Figure1b: The Shuttle Campaign Model II (Ejembi, 1989) 


\section{Target Audience Conditions that Impact on Graphic Language}

Among many factors that affect the graphic encoder and invariably, the graphic language employed to share development messages, illiteracy, and crosscultural variables are key. The adequate consideration of the extent, dimensions and implications of these are pivotal for success in the transformation process of development ideas into graphic language that suits target audience information need.

\section{The Illiteracy Factor}

Given the high proportion of non-literates in the developing world (over $80 \%$ in some areas), graphic language must be specially adapted in order to provide an effective means of communication with target audience. This is challenging because the process of mobilizing, training, and educating manpower for development purposes requires the use of language codes, which are in most cases, above the elementary level of literacy. The challenge is even more complex as skills such as visual perception and scientific thinking are neglected at the expense of literacy and numeracy in African primary schools. In the above citation, Ejembi (1989: p 108), stressed the need for the adaptation of development ideas into suitable graphic language for target audience. This is particularly critical in development communication campaign in Africa, where the high rate of ignorance, superstition, and illiteracy are aggravated by lack of training in scientific thinking, and visual perception.

Although, literacy rate in most of the developing world has risen from over $20 \%$ in 1970 to $70 \%$ in 2019, much work still need be done (Tasamba, 2019). Likewise, Shiunde (2018: p 33), sounded a cautionary note from the Human Development Report (2016) of the United Nations Development Programme (UNDP), which stresses that "while many countries have made gains in access to education, improvement in the quality of education have not kept pace". Quality education need impart the capacities to think critically, being creative, communicating effectively, and cooperating with others as well as being computer, information, and media literate. This is salient to meet $21^{\text {st }}$ century demands. In addition, Shiunde (ibid), citing the United Nations Educational, Scientific and Cultural Organization (UNESCO) Factsheet No. 45, September, 2017 , states that "it is worrisome therefore, that $27 \%$ of the world's illiterate people live in sub-Saharan Africa; and 17 countries in Africa still have literacy rates of $50 \%$ and below.

The above is a major reason for a large number of the target audience still experiencing difficulty in grasping graphic communication in developing nations. Ejembi (1989, p: 111) citing Zimmerman and Perkin (1982, p.5) emphasized the relative difficulty to finding appropriate combination of words and pictures for receiver with largely unknown abilities of pictorial perception. So, it is needful to evaluate the quality and effectiveness of words/pictures in a given situation by identifying the difference between elements, structures and functions of words/pictures and how these impact on communication in a given context and culture. Also, it is paramount to interpret the socio-cultural context in which words/pictures would be utilized, and the instructional event that makes up 
development communication. This is to avoid the prevalent communication failure, which is due to certain aspects of graphic communication taking for granted because they are established conventions in the Western world.

Nevertheless, efforts have been made by nations, organizations and researchers (for example, Nigeria 6, 3, 3, 4 education policy, UNESCO, and Paulo Freire, respectively), to eradicate illiteracy at basic, proficiency, advanced, or functional levels. While Basic literacy avails the non-literate with adequate reading, arithmetic and writing skills (through the school system of primary, secondary, tertiary; mass campaign, continuing adult basic education, and elearning), Functional literacy provides problem-solving and critical thinking skills to insufficiently utilized manpower through training, mobilizing, and educating for higher productivity and usefulness to self and society. Functional literacy adopts two approaches that have immense implication for the use of graphic language in development communication. The first, is the Extension strategy, adopted by UNESCO, whereby target audiences are imparted with skills from an advance source, taking into consideration the socio-cultural environment, though information flow is from above and outside. The communication practice deployed is the dominant/hierarchical model. The second approach is the Freirian doctrine of 'Conscientization', whereby key words/pictures are employed to stimulate target audience to think critically about their existing depraved conditions and generate actions to occasion change. This strategy adopts the development paradigm of Multiplicity, which is characterized by information flow from within and below, employing the participatory horizontal communication practices.

The above pinpoints two areas where necessary knowledge have been produced to decisively use graphic language for human development programmes, these are visual literacy and perception studies. Visual literacy is the ability to grasp and interpret pictures, while perception is the competence to recognize, select, organize, and interpret meanings expressed in visual stimuli. Ejembi (1989, pp. 118), in summarizing researches on visual literacy and cross-cultural studies, stated two reasons for perception of pictorial materials thus: (1) pictorial communication is best in sharing messages with non-literate receivers in crosscultural environment; and (2) pictorial communication is critical to illustrating and explaining complex ideas that are often difficult to express by words alone; which is in accord with the adage 'a picture is worth more than a thousand words'. Nonetheless, it is worth stating at this juncture that words and pictures are meaningless to a receiver who cannot grasp them.

\section{The Cross-Cultural Factor}

Concerted efforts of communication for development do occur in a sociocultural environment, which is made up of a distinctive body of knowledge, attitudes, skills, languages, and experiences from other ways of life. This affords recognition, production, and interpretation of a set of symbolic codes and modes of expression in identical fashion. So, when development messages meant for a particular culture is deployed in another, problems of interpretation are bound to emerge. Similarly, there is problem of interpretation of pictorial representation when different cultures, as it is in Nigeria (Hausa, Ibo, Yoruba, Ijaw and 273 
dialects) share a common language, English. Ejembi (1989, p.119) states that with this plurality of dialects spoken across cultural boundaries in Nigeria, uniformity of interpretation of messages encoded in a particular graphic language is difficult. Therefore, producing and using information, education and communication materials with western conventions to mobilize, train and educate non-western target audience without proper evaluation is counter-productive. This situation is aggravated by the urgency that usually accompanies development communication campaigns, and accounts for the high rate of pictorial communication failure.

Furthermore, the obvious and non-obvious aspects of graphic elements in a cross-cultural context is a dimension that presents a daunting challenge. The meanings given to words in different languages are often clear and distinctive. On the contrary, interpretations given to pictorial symbols do carry connotative meanings that are often different from one culture to another. Ejembi (1989), aptly illustrated this in a graphic model (see figure 2).

CROSS-CULTURAL PROBLEMS:

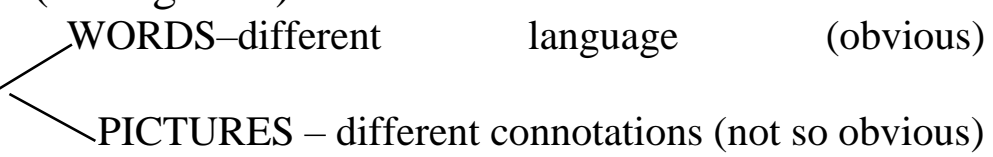

Figure 2: Showing obvious and non-obvious aspects of graphic language elements in a crosscultural context (Culled from Ejembi, 1989. P.108)

From the foregoing, it is reasonable to state that different culture produce different pictures. Also, different cultures perceive visual expressions differently. In addition, some cultures, as could be seen from early Egyptian drawings for example, have problems with depth perception following western conventions. And it is obvious that perception of pictorial representations are impacted on by the influence of knowledge and experience. As a result, when producing pictorial stimuli for a given target audience, it is important to be aware of the ambiguities and inadequacies that are bound to occur during the encoding process of development ideas into graphic language; and ensure that aesthetic or arousal, pragmatic, semantic, syntactic, and technical uncertainties are satisfactorily resolved. This is indispensable because the content, form, and attributes of a given message affect the way it is perceived and responded to by target audience. This brings us to the nature of stimulus materials and response elicited in a given sociocultural environment.

The nature of stimulus materials consist of its compositional variables (elements of design structured in accord with organizational principles). This could be standard/simplified depending on the level of interaction the source allows during the media development process. The source could import foreign, standard graphic language strictly developed and considered appropriate for sharing messages by experts without much interaction with, and real intervention of receivers, which is the case in Modernization and Dependency paradigms of development. Conversely, the source could permit a high degree of interaction between all communication actors including the receiver, instead of sole reliance on experts in the media production. This is the case in Another Development or Multiplicity concept of development and often result in the production of suitable simplified graphic language.

Obviously, to achieve desired response from the receiver, it is needful to 
create stimulus conditions that align with target audience conditions. Towards this end, Cook (1981), and Zimmerman and Perkin (1982), provided some guidelines, recommendations or rules of thumb for creating simplified pictorial composition thus: pictures should be configured to be realistic or representational, having resemblance to its referent (e.g. realistic drawings, illustrations and photographs). It should depict familiar subject matter, symbols and these should be kept simple with essential details that are not excessive or scanty. This affords analogous pictorial configuration, having superficial appearance or general surface contour of its referent with nuances or details largely omitted but basically allows identification to a great extent (e.g. silhouette of a person or an object, and high focus line drawings). Moreover, background of pictures should adequately contrast with figures; and photographs should be used where illustrations are inadequate. In addition, art, text and copy should be balanced; and pictorial sequence ordered chronologically. Contrarily, graphic language should not be configured in arbitrary format, having no resemblance to its referent (e.g., abstract drawing/illustration, charts, graphs, and maps). Finally, pictorial composition should be pretested for target audience to have a final say about content, illustration and sequencing; and materials for national distribution should take into account differences in interpretation of pictorial messages across regional/cultural boundaries.

Although, the above prescribed ways to configure the elements and structures of pictorial composition in order to achieve desired response from target audience for persuasion, or instructional purpose, are useful guidelines but not rules. However, there are no hard and fast rules for the graphic encoder, who should be a graphic design specialist (in this paper, specialist refers to a person, who has gone through a university, polytechnic or graphic design training programme). In creating the appearance/nature of form for development communication, the graphic encoder is technically equipped with skills and formgeneration principles/theories to proffer effective design solutions to meet communication challenges, especially when embedded in the entire media production process. Thus, the graphic encoder is positioned to effectively mediate between the source's ideas and receiver's information need. Nevertheless, the three aforementioned nature of graphic language (representational, analogous, and arbitrary) could be found in stock, clip, and vector art and readily adopted or adapted for use as standard or simplified graphic language respectively. A rash decision made on this, often results in ineffective graphic media that is inimical to achieving desired communication goal. This brings us to the use of standard or simplified graphic language for development communication campaigns in Africa.

\section{Use of Standard/Simplified Graphic Language in Campaign}

The ORT, Delayed Sexual Debut and Use Condom, are three development communication campaigns in Africa that could be employed to exemplify the use of standard or simplified graphic language and response elicited from target audience in developing countries.

\section{The ORT Campaign in Egypt}

The logo development for the Oral Rehydration Therapy (ORT) Campaign in 
Egypt is a lucid analogy of the decision to use standard graphic language, which appealed to the source, who selected and deemed it fit for the intended receivers. This was found unsuitable and was changed to simplified graphic language by the intervention of the target audience during the media development process. The first Executive Director of the Egypt ORT Project was to decide on and adopt a logo for the project. Having examined many logo ideas submitted to the project by different artists and designers, he liked one, which is arbitrary or abstract and stylized with minimal basic shapes and lines that are not definitive but suggestive of the action of a mother giving her child the ORT solution. This mode of presentation is the prevalent feature of imported standard graphic language, and largely appeals to a more visually educated persons, who could understand and interpret them. Understandably, the director, preferred the abstract design more than the others and was tempted to adopt it for the project without further deliberation (see Figure 3a). However, he decided to withhold his opinion until all logos had been tested.
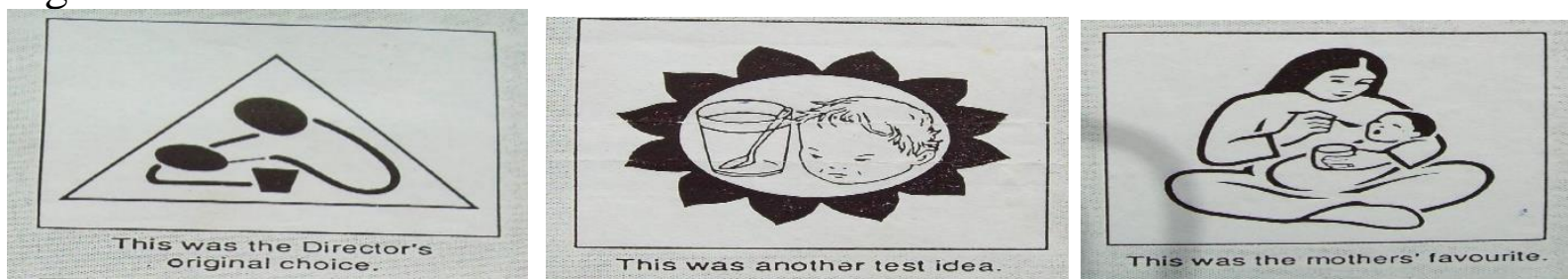

Figure 3: Logos Pre-tested for the ORT Campaign in Egypt, showing how test improved logo

(Culled from WHO, 1987)

When the logos were tested with a sample of the target audience (mothers), the result indicates that the logo the director preferred was the one least favoured by the pre-test respondents. The mother's choice was in representational mode of graphic language, simplified, with lines that clearly described in detail the appearance of the mother, spoon and cup containing the ORT solution, attire, and the child in a style they are familiar with. Also, the sample tested, pointed out that their choice of logo needed to be revised in order to be more effective. Again, a large number of the respondents opined that the colour be modified and the mother pictured in the logo should wear a wedding ring. However, the logo selected by the pre-test respondents was adopted for the ORT campaign. The experience so impressed the first Executive Director that he often recounted the story, to emphasise the need to pre-test draft with target. Had the director adopted the particular logo he liked without the target audience involvement in the development process through pre-test, the necessary and important improvements made on the logo, would have been missed. The introduction of a wedding ring on the hand of the pictured mother in the logo, is paramount and instructive. It is a staple of the socio-cultural influence on the interpretation and acceptance of graphic language.

Significantly, it points to the socio-cultural interpretation of motherhood as a product of legality in marriage, symbolised by the wearing of a wedding ring. It also points to the fact that very crucial point can be omitted when making initial draft, which could be corrected during the pre-test and evaluation process. The omissions, if not corrected can significantly affect target audience response. The above shows the need for graphic media to be thoroughly pre-tested with target 
audience and recoded (if necessary) before final production and distribution are made. The intended receiver's knowledge, attitude, beliefs, perception and response are revealed during pretest. From this standpoint of view, informed decision on the use of standard or simplified graphic language for communication with the target audience could be taken decisively.

\section{The Delayed Sexual Debut Campaign in Nigeria}

The Delayed Sexual Debut Campaign in Nigeria was a rare success. Its utilization of simplified graphic language is another vivid example of informed decision-making on the basis of target audience evaluation. In 2004, the Delayed Sexual Debut Campaign, with 'Zip Up' catch phrase, and tagged 'Make We Talk' was launched. This was due to the urgency to stem the high rate of death from AIDS, sexually transmitted diseases, and teenage pregnancy. The aim was to encourage delay of sex until marriage and increase the median age of sexual debut. The campaign was part of a seven year (2002-2008) project 'Promoting Sexual and Reproductive Health for HIV/AIDS Reduction. PSRHH Programme, was funded by the British Department for International Development (DFID) and the United States Agency for International Development (USAID). The media production process of the campaign started with desktop research that analysed the existing situation, and provided a manual 'Creative Brief', which stated the source's aim and target audience. Then, the graphic encoder (LTC-JWT Advertising Limited) generated media drafts that were pre-tested with sample of the target audience and necessary adjustments were made. Taking audience conditions into consideration, fashion trends and commonly spoken language in the North (Hausa), and in the South (English/Pidgin), were used (see figure 4a, b, $\mathrm{c}$, and d).

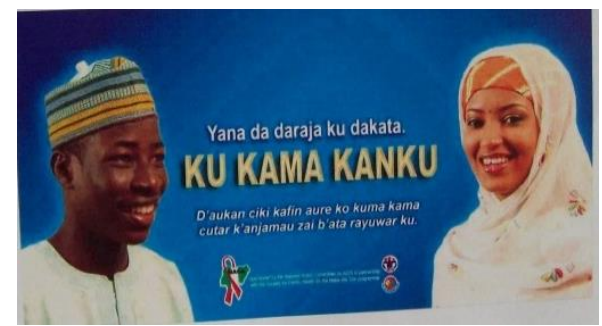

a

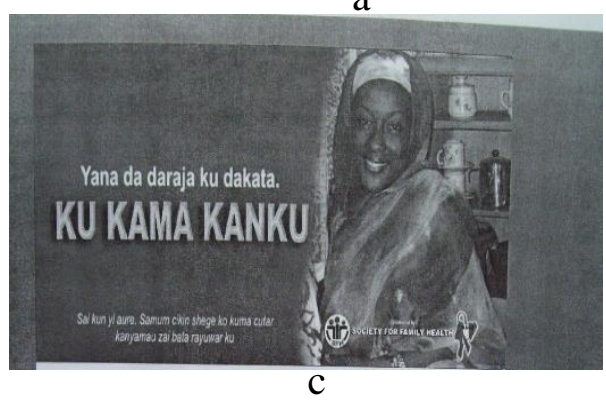

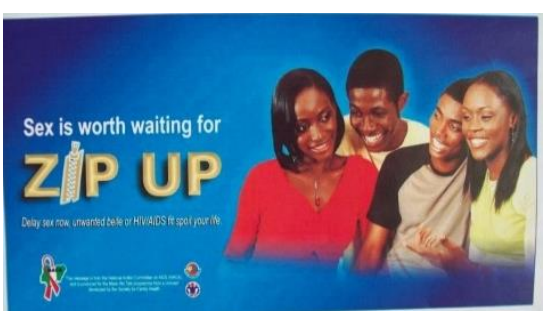

b

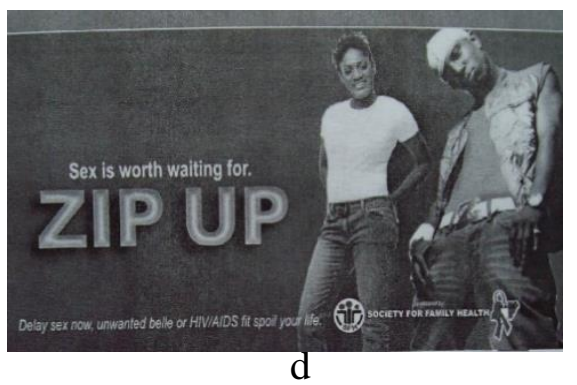

Figure 4 (a, and b): Billboards in Hausa, and English/Pidgin versions for North, and South respectively; (c and d): Television Spots in Hausa, and English/Pidgin versions for North and South respectively, produced by the Society of Family Health (Culled from Ebigbagha, 2016)

The billboard posters and television spots are encoded decidedly with simplified graphic language, which showed trendy cloths 'MTV Style' that are fashionable, attractive, and not obscene. Thus, emphatically expressing coalesce of trendiness and decency without obscenity. The certainty of using simplified 
graphic language for the campaign was hinged on target audience evaluation and intervention that occasioned success. It scored indelible marks in the heart of receivers and made 'Zip Up' a popular response catch phrase (which means 'let us delay sex instead of having it now') among target audience (SFH, Corporate Presentation, 2006).

\section{The Use Condom Campaign in Nigeria}

The Use Condom Campaign in Nigeria was launched by the Society for Women and Aids in Africa Nigeria (SWAAN) and funded by World Health Organisation, DFID and USAID. It is a typical example of concerted communication efforts of prevalence use of Standard graphic language to share development messages with target audience. Although, the graphic media employed passed through many stages of the media production process including preparatory workshops that involved stakeholders. Also, steps were taken to make clarification with the source's aim and target audience, topic study, pre-test and evaluation. However, the source directed the entire communication development process. The pre-test of the media draft was carried out with experts from the country of the funding agencies instead of the target audience (Ebigbagha, 2016). The neglect of the target audience intervention, and the proper mediation role of the graphic encoder between the development ideas of the source and information needs of the target audience, resulted in standard graphic language that was strange to the intended receivers. The graphic message was fraught with aesthetic or arousal, pragmatic, semantic, syntactic, and technical uncertainties (see figure $5 a$ and $b)$.
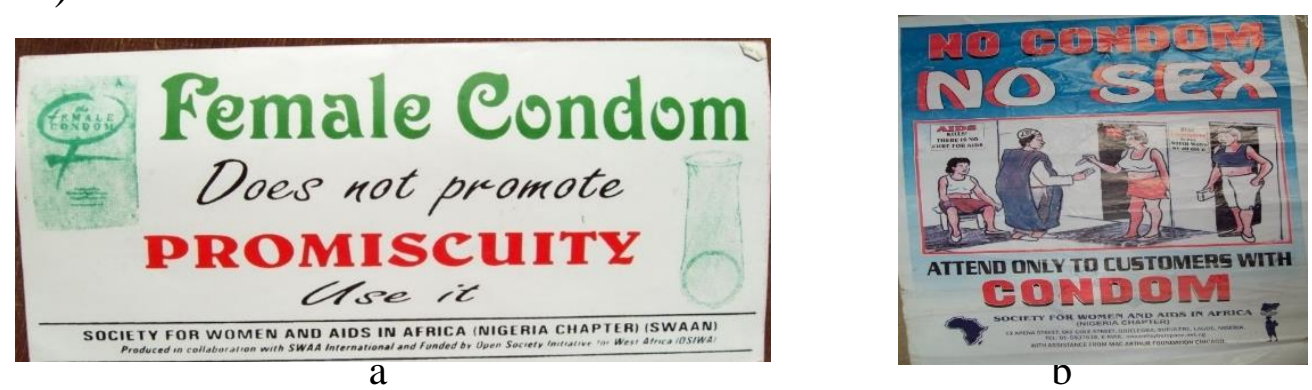

Figure 5a and b: A Sticker on 'Female Condom Does not Promote Promiscuity, Use it'; and a Poster on 'No Condom, No Sex' Produced by SWAAN (Culled from Ebigbagha, 2016)

In the sticker, the word 'Promiscuity' is emphasised with red colour and boldly written in capital letters. The prime message 'Female Condom' is deemphasised with green colour, though boldly written in capital and low letters. The supporting information 'Does not promote' and 'use it' are written in slim italics and further deemphasised with black. The pictorial image of the condom, and its packet, are placed on both sides and further deemphasised with green colour in half tone. Each set of words are expressed in four different typefaces. Generally, the haphazard placement of both graphic and typographic elements defies any existing prescribed layout for the production of graphic language. At a distance, only three words 'Female Condom Promiscuity' could be clearly seen. The graphic language seem to encourage promiscuity, which is against its desired communication goal. Aside, it is unattractive, uninteresting and loaded with uncertainties. The iconic images were so pale and unimpressive that target audience asked for live representation for the purpose of clarity because they could 
neither understand it nor identify what it was. It lacks all the qualities of been handled by experts who are specialist in design, which could have been improved upon had it been pre-tested with receivers. It did not elicit the desired response from the target audience, so it was a communication failure.

Likewise, the configuration of the poster was a misleading misrepresentation. Its main aim was to warn against unprotected sex, and promote the use of condom as a measure to stem HIV/AIDS. It depicted a man in an attire worn by western and northern Nigerians, interacting with one of the three prostitutes, each in front of a door to a room in a brothel. The illustration is fair but contains elements that are inimical to the campaign because of semantic uncertainties. Some interpretations that could be inferred from it are: (i) only those who go to brothel need use condom to avoid contracting HIV/AIDS. (ii) Only those who patronise prostitutes need use condom to avoid contracting HIV/AIDS. (iii) Only those who are from the western and northern parts of Nigeria need use condom to avoid contracting HIV/AIDS; and (iv) Only those who want to prevent HIV/AIDS need use condom. The above would have been resolved had the poster been pre-tested with target audience. The attire of the man could have been revised because of its socio-cultural implication. Also, the attention to brothels and prostitutes would have been modified. This is because the most liable age group (fifteen to twenty-five years) to HIV/AIDS, are more in schools and on streets than in brothels. In spite of the fact that the graphic language in the sticker and poster were not pre-tested with target audience, the inability of the target audience to identify, recognise and correctly interpret the female condom in the sticker and asked for live presentation was instructive.

It underscores the need to use only familiar objects with target audience, and where this is not possible, media that could depict 3dimensional, and 4dimensional (space-time continuum) experiences should be considered. This is due to the inherent limitation of pictorial composition, which can only represent experiences in 2dimensional mode using flat cues. From the foregoing, the Use Condom campaign was a communication failure. This was largely due to rash decision-making on the graphic language suitable for a given target audience before it was mass produced and distributed.

\section{Finding}

The paper found that pre-testing media draft with target audience is indispensable for informed decision-making on whether to use standard or simplified graphic language for a given target audience. This happens when the source, the graphic encoder and the target audience perform their roles/activities properly during development of the graphic language at its preparatory and advance stages in a communication campaign. Moreover, a high-level interaction/collaboration between communication actors, ensures use of graphic language with salutary effect as epitomised by the ORT, and Delayed Sexual Debut Campaigns. While, low-level intervention/collaboration culminates in rash decision-making on the use of graphic language and communication failure as seen in the Use Condom Campaign. 


\section{Conclusion}

A key panacea for the communication challenge to decidedly use standard or simplified graphic language in support of development initiatives, is target audience-based pretest. This largely ameliorates constraints resulting from illiteracy and perceptual differences from cross-cultural boundaries prevalent in developing nations. It enables transformation of the source development ideas into graphic language that takes into account target audience conditions with salutary effect. The above is impeded by adoption of hierarchical communication practices as in Modernization or Dependency concepts of development, which allows the source to lead the entire media development process at the expense of other stakeholders. Conversely, it is fostered by employing horizontal communication practices as in Multiplicity paradigm of development that afford effective collaboration and participation of communication actors. This practices should be adopted to make informed decisions on graphic language for prevalent non-literate receivers and realize success in development communication campaign.

\section{References}

1. Cook, B. L. (1981), Understanding pictures in Papua New Guinea: What kind of pictures communicate most effectively with people who cannot read? David C. Cook Foundation, Elgin

2. Ebigbagha, S. Z. (2016), Graphic Encoding Choices in Development Communication: Identifying the Several Loops of the Graphic Encoder/Media Team Collaboration. Lap Lambert Academic Publishing, Germany

3. Ebigbagha, S. Z. (2020), 'The Graphic Communication Actor: Generating Visual Rhetoric for Development Initiatives. Sciendo, Editura Artes, Romania. Volume XVI Pp. 94-106

4. Ejembi, E.A. (1989), Graphic Communication in Development Programmes: Models for Communicators. An Unpublished Ph.D. Thesis, Department of Typography and Graphic Communication, University of Reading, England

5. McQuail, D. and Windahl, S. (1986), Communication Models for the Study of Mass Communication. Longman, London, England

6. Obionu, C.N. (2001), Primary Health Care for Developing Countries. Delta Publications (Nigeria) Limited, Enugu, Nigeria

7. Shiunde, A. (2018), Literacy Rates have Gone up in Sub-Saharan Africa. e.paper no. 9, 2018/09 p.33. https://twitter.com/Shiunde. Retrieved; 30/9/2020.

8. Society of Family Health (2006), Corporate Presentation. Society of Family Health, Abuja, Nigeria

9. Tasamba, J. (2019), African Countries Push for Higher Literacy. https://www.dandc.eu>articles >literacy-ra. Retrieved: 2/10/2020

10. World Health Organisation (1987), Communication - A Guide for managers of National Diarrhoeal Disease Control Programmes. World Health Organisation, Geneva, Switzerland

11. Zimmerman, M. L. and Perkin, G. W. (1982). Print Materials for Nonreaders: Experiences in Family Planning and Health. Program for the Introduction and Adaptation of Contraceptive Technology (PIACT). Paper 8 